\title{
Relationship between exercise-induced heart rate increase and the formation of microbubbles and high-intensity transient signals in mechanical heart valve implanted patients
}

Ayșegül Sünbül ${ }^{1}$, Ahmet KIrbaș², Nursen Tanrlkulu³, Cihan Șengül ${ }^{4}$, Bahadır Dağdeviren5 Ömer Ișlk²

1Department of Cardiology, Medicana Camlica Hospitals, İstanbul, Turkey

2Department of Cardiovascular Surgery, Medicana Camlica Hospitals, İstanbul, Turkey ${ }^{3}$ Department of Anesthesiology and Reanimation, Medicana Camlica Hospital, İstanbul, Turkey

${ }^{4}$ Department of Cardiology, Gaziosmanpa a Hospital, İstanbul, Turkey

${ }^{5}$ Department of Cardiology, Medical Park Hospital, İstanbul, Turkey

Submitted: 2 March 2012

Accepted: 24 April 2012

Arch Med Sci 2014; 10, 4: 701-705

DOI: 10.5114/aoms.2013.34990

Copyright $\odot 2014$ Termedia \& Banach

\author{
Corresponding author: \\ Ahmet Kırbaș \\ Department \\ of Cardiovascular Surgery \\ Alemdag cad. No: 85 \\ 34767 istanbul, Turkey \\ E-mail: \\ ahmetkirbas@gmail.com
}

\begin{abstract}
Introduction: The formation and collapse of vapor-filled bubbles near a mechanical heart valve is called cavitation. Microbubbles can be detected in vivo by doppler ultrasonography (USG) as HITS (high intensity transient signals) in cranial circulation. We investigated the relationship between exercise induced heart rate increase and HITS formation in cranial circulation.

Material and methods: Thirty-nine mechanical heart valve implanted ( 8 aortic valve replacement (AVR) + mitral valve replacement (MVR), 9 AVR, 22 MVR) patients aged 18-80 years old were included in our study. Microbubbles were counted in the left ventricular cavity via transthoracic echocardiography at rest per cardiac cycle. Afterwards transcranial Doppler USG was performed and HITS were counted in each patient's middle cerebral artery at 5 min duration. Subsequently an exercise test according to the Bruce protocol was performed. After achieving maximal heart rate, microbubbles in the left ventricle and HITS were counted again.

Results: Microbubbles in the left ventricle and transcranial HITS increased after exercise significantly compared to resting values $(15.79 \pm 10.91$ microbubbles/beat vs. $26.51 \pm 18.00$ microbubbles/beat, $p<0.001 ; 6.13 \pm 8.07 \mathrm{HITS} / 5 \mathrm{~min}$ vs. 13.15 $\pm 15.87 \mathrm{HITS} / 5 \mathrm{~min}, p=0.001)$. There was a significant correlation between microbubbles and HITS counts after peak exercise $(r=0.55, p<0.001)$.

Conclusions: In our study, we found that the microbubbles were increasing as the heart rate increased and more HITS were propelled to the cerebral circulation. As previously shown, HITS can alter cognitive functions. Therefore heart rate control is essential in mechanical heart valve patients to protect neurocognitive functions.
\end{abstract}

Key words: microbubble, high-intensity transient signals, transcranial, mechanical heart valve, exercise.

\section{Introduction}

In the late 1960s, decompression bubbles in divers were described by means of diagnostic ultrasound as well as arterial aeroembolism during cardiac surgery. These clinically silent signals, preferably called high-inten- 
sity transient signals (HITS), are detectable within the transcranial Doppler (TCD) frequency spectrum $[1,2]$. The brief transient increases in the intensity of the reflected ultrasound, designated as HITS, represent the TCD signatures of the presence of air or solid emboli in the cerebral circulation [3, 4].

In patients with MHVs, the frequency of HITS depends on the type of valve installed, whereas the prevalence of HITS is much lower with bioprostheses. The majority of HITS, particularly in MHV carriers, reflect gaseous microemboli due to cavitation at the rim of the MHV and not to the shedding of thromboembolic material. It is not clear whether HITS are induced by solid elements such as thrombotic aggregates or gas bubbles. The latter are generated under hemodynamic stress due to turbulent blood flow at the valvular level [5]. These turbulences are caused by cavitation in areas of high sheer stress and high pressure gradients [6, 7]. Closure dynamics of MHV often trigger a cascade of events, which are instigated by an abrupt and transient reduction of local pressure. This results in cavitation in the blood [8] on pre-existing nuclei, followed by growth of persistent gas-filled microbubbles. This hypothesis is supported by the stable number of HITS found during repeated investigations and the lack of patients' clinical deterioration over time. Also, the rate of cardioembolic stroke in these patients does not appear to be correlated with the number of HITS detected. No correlation could be identified between the patient's hemostaseology and either the number of HITS or the type and extent of antithrombotic treatment [9].

The aim of this study was to determine the relationship between exercise-induced heart rate increase and the formation of microbubbles and HITS in mechanical heart valve implanted patients.

\section{Material and methods}

All data were collected on a prospective basis. Prior to inclusion of patients in the study, informed consent was obtained. The study was conducted according to the Helsinki Declaration and approved by the Ethics Committee of our hospitals. Thirtynine mechanical heart valve implanted (mitral and aortic position) patients aged $18-80$ years old were included in our study. We selected subjects with mechanical prosthetic heart valves, according to the following criteria: I - normally functioning valves; II - bright echoes suggesting microbubbles, inside the prosthetic valve and/or upstream from it (left ventricular outflow tract or left atrium); III no morphological lesions that could generate solid microemboli at the prosthetic level; IV - normal carotid arteries, as investigated by color echoDoppler; V - therapeutic INR levels.

Transthoracic echocardiography was performed using the GE vivid 7 dimension 5-7 MHz probe.
Echocardiographic examination was performed to validate the ejection fraction, valvular pressure gradient, left ventricle end diastolic dimension, left ventricle end systolic dimension, left atrial size, microbubbles in left ventricle per cardiac cycle, and prosthetic heart valve (mitral position) m-slope views. Microbubbles were counted in the left ventricular cavity per cardiac cycle. Then transcranial Doppler USG was performed using the GE vivid 7 dimension $5 \mathrm{MHz}$ probe. The patient was placed in the supine position and 5-MHz ultrasound transducers were attached temporally over the pre-auricular transcranial window. Transcranial HITS were counted in each patient's middle cerebral artery at five minutes duration. Subsequently an exercise test according to the Bruce protocol was performed. After achieving maximal heart rate, microbubbles in left ventricle per cardiac cycle and HITS were counted again for five minutes duration.

\section{Statistical analysis}

Continuous (quantitative) numerical values were expressed with the mean \pm standard deviation, and categorical (nominal) values. To compare continuous variables paired Student's $t$ test was used. Value of $p<0.05$ was considered as statistically significant. Pearson correlation analysis was performed to assess the relationship between continuous variables.

\section{Results}

Twenty-one of 39 patients in our study were male and 18 female. The average age of the patients was $54.0 \pm 13.7$ years. The average resting heart rate was $74.4 \pm 15.1$, and average heart rate after exercise was $122.9 \pm 25.27$. Nine of 39 patients had aortic valve replacement and 30 of 39 patients had mitral valve replacement. Seventeen patients had sinus rhythm and 22 patients had atrial fibrillation rhythm. At rest per cycle within the left ventricle there were $15.7 \pm 10.7$ microbubbles. After exercise per cycle within the left ventricle there were 26.5 \pm 18.1 microbubbles. At rest during five minutes transcranial Doppler detected $6.1 \pm 8.1$ HITS. After exercise during five minutes transcranial Doppler detected $13.1 \pm 15.8$ HITS (Table I). The value of 0.488 for the relationship between resting heart rate and post-exercise heart rate is positive and statistically significant $(r=0.488 ; p=0.002)$. The value of 0.903 for the relationship between resting microbubbles and post-exercise microbubbles is positive and statistically significant $(r=0.903 ; p=0.0001)$. The value of 0.943 for the relationship between resting HITS and post-exercise HITS is positive and statistically significant $(r=0.903 ; p=0.0001)$. The value of 0.879 for the relationship between resting mitral valve closure slope and post-exercise mitral valve closure slope is positive and statistically sig- 
Table I. Demographic data

\begin{tabular}{|lccccc|}
\hline Parameter & Number & Min. & Max. & Mean & SD \\
\hline Age & 39 & 27 & 82 & 54.5 & 13.7 \\
\hline LV EF & 39 & 40 & 60 & 53.25 & 6.1 \\
\hline LVEDV & 39 & 55 & 180 & 109.64 & 34.38 \\
\hline LVESV & 39 & 24 & 110 & 52.51 & 19.14 \\
\hline LVEDD & 39 & 3.90 & 6.7 & 5.1 & 0.65 \\
\hline LVESD & 39 & 2.4 & 4.8 & 3.5 & 0.6 \\
\hline LA AP diameters & 39 & 3.0 & 6.15 & 4.7 & 0.75 \\
\hline Resting heart rate & 39 & 50 & 130 & 74.4 & 15.17 \\
\hline Post-exercise heart rate & 39 & 80 & 180 & 122.9 & 25.27 \\
\hline Resting microbubbles & 39 & 2 & 57 & 15.79 & 10.91 \\
\hline Post-exercise microbubbles & 39 & 5 & 90 & 26.51 & 18.00 \\
\hline Resting HITS & 39 & 0 & 36 & 6.13 & 8.06 \\
\hline Post-exercise HITS & 39 & 0 & 56 & 13.15 & 15.87 \\
\hline Resting mitral valve closure slope & 17 & 16 & 82 & 41.94 & 17.37 \\
\hline Post-exercise mitral valve closure slope & 17 & 35 & 162 & 71.87 & 32.63 \\
\hline
\end{tabular}

LVEF - left ventricle ejection fraction, LVEDV - left ventricle end diastolic volume, LVESV - left ventricle end systolic volume, LVEDD - left ventricle end diastolic diameter, LVESD - left ventricle end systolic diameter, LA AP - left atrium anteroposterior. Data are given as mean \pm standard deviation or $n(\%)$, where applicable

nificant $(r=0.943 ; p=0.0001)$ (Table II). The value of 0.502 for the relationship between resting microbubbles and post-exercise HITS is positive and statistically significant $(r=0.502, p<0.05)$. The value of 0.961 for the relationship between resting HITS and post-exercise HITS is positive and statistically significant $(r=0.961, p=0.0001)$. The value of 0.552 for the relationship between post-exercise microbubbles and post-exercise HITS is positive and statistically significant $(r=0.552, p=0.0001)$ (Table III).

\section{Discussion}

High-intensity transient signals were initially observed by cardiac surgeons during operations using ECC [10]. There are numerous studies indicating a correlation between HITS during ECC and neurocognitive dysfunction [11]. The frequency of microemboli in patients with heart disease varies considerably, with the highest prevalence and frequency in patients with mechanical heart valves (MHVs). These patients may have several hundred microemboli per hour without experiencing clinical symptoms [12]. We did not perform any questionnaire about neurocognitive defects in this study.

We investigated the relationship between exercise-induced heart rate increase and the formation of microbubbles and HITS in mechanical heart valve implanted patients. The value of 0.502 for the relationship between resting microbubbles and post-

Table II. Correlation analysis between resting and post-exercise parameters

\begin{tabular}{|llcc|}
\hline Variables pairs & $N$ & Correlation & Value of $p$ \\
\hline Resting heart rate \& post-exercise heart rate & 39 & 0.488 & 0.002 \\
\hline Resting microbubbles \& post-exercise microbubbles & 39 & 0.903 & 0.0001 \\
\hline Resting HITS \& post-exercise HITS & 39 & 0.943 & 0.0001 \\
\hline Resting mitral valve closure slope \& post-exercise mitral valve closure slope & 17 & 0.879 & 0.0001 \\
\hline
\end{tabular}

Table III. Bivariate correlation analysis between resting and post-exercise parameters

\begin{tabular}{|lccc|}
\hline Variables pairs & $N$ & Correlation & Value of $p$ \\
\hline Resting microbubbles \& post-exercise HITS & 39 & 0.502 & 0.001 \\
\hline Resting HITS \& post-exercise HITS & 39 & 0.961 & $<0.001$ \\
\hline Post-exercise microbubbles \& post-exercise HITS & 39 & 0.552 & $<0.001$ \\
\hline
\end{tabular}


exercise HITS is positive and statistically significant $(r=0.502, p=0.0001)$. The value of 0.961 for the relationship between resting HITS and post-exercise HITS is positive and statistically significant $(r=0.961, p=0.0001)$. Gencbay et al. found a strong correlation between serum lactic dehydrogenase (LDH) levels and quantity of bubbles. There was a strong correlation between serum LDH levels and microbubble counts, which suggests that hemolysis may be related to microbubble formation [13]. Microbubbles that are not fragmented participate in the cerebral circulation as HITS.

The origin (up- or downstream from the valve) and nature (gaseous or solid) of HITS remain unclear. A clear correlation between HITS and the hemodynamic performance of the valve has also not yet been proven, as HITS can occur independently of valve size, hemodynamic parameters and patient characteristics [14]. High-intensity transient signals are commonly reported in the cerebral circulation of patients with MHVs [14]. The embolic signals recorded from these patients have been described as being more intense than those found in patients with other potential embolic sources and thus are likely to be due to large solid emboli or gaseous bubbles that have a high ultrasound reflectivity. Several arguments have been made in favor of a gaseous origin of HITS associated with MHV and have been previously discussed. Among these arguments, the lack of relationship between the degree of anticoagulation, antithrombotic, or antiplatelet therapy and the HITS rate must be highlighted. Moreover, microembolic signals are not observed in normally functioning biological valves. A low HITS rate has however been reported in patients with biological prostheses. It must be stressed that in these reports there was no valve or carotid control, and the microemboli observed could be of a solid nature. Oxygen inhibits the cavitation process of mechanical prosthetic heart valves or speeds up redissolution of gas bubbles generated by cavitation. Cavitation, i.e., formation of bubbles from a gas dissolved in the blood, in patients with mechanical prosthetic cardiac valves is a well-known phenomenon. It was found that cavitation is generated primarily by the deceleration of the closing body of the valve. In this study we found a relationship between resting mitral valve closure slope and post-exercise mitral valve closure slope. According to this, mechanical valves oriented in a mitral position may process more microbubbles and HITS. The pressure drop produced thereby is overlapped by the pressure drop in accelerated or turbulent flow regions produced by design characteristics at outlet struts, stop faces, or sealing lips during backflow through the closing disk. Smaller valves have higher cavitation thresholds than larger ones. Some of these bubbles persist and are washed out into the cerebral circulation where they can be detected by TCD. In another study after oxygen inhalation the number of HITS decreased [15].

In previous in vivo studies the influence of valve orientation on the hemodynamic performance of mechanical aortic valves has been demonstrated for the tilting disc and bileaflet valve design. In these studies the optimally oriented Medtronic Hall valve (major orifice facing the non-coronary leaflet) showed superior results especially with respect to downstream turbulence over the bileaflet valve in any orientation. As downstream turbulence leads to platelet activation, the number of microemboli originating from a tilting disc valve might also be influenced and this might explain the superior results of the tilting disc valves regarding microembolization [14].

Deklunder et al. investigated the effects of myocardial contractility and heart rate on microemboli production in association with conventional and experimental mechanical heart valves implanted in the mitral position in a bovine model. In 10 calves, the mitral valves were replaced with mechanical valves. Doppler recordings were analyzed for highintensity transient signals, which are ultrasound reflections from circulating microemboli. The animals were studied at rest, during pacing at $160 \mathrm{bpm}$, after dobutamine infusion, and after esmolol infusion. The incidence of high-intensity transient signals was expressed as signal frequency (signals per hour) and as signal rate (signals per 100 heart cycles). With a $68 \%$ increase in the heart rate, signal frequency increased by $135 \%$, but signal rate increased by only $41 \%$. With a $144 \%$ increase in myocardial contractility, signal rate increased by $264 \%$. With a $31 \%$ decrease in contractility, signal rate decreased by $62 \%$. They concluded that microemboli production by mechanical heart valves varies with myocardial contractility and heart rate. The fact that contractility affects the incidence of highintensity transient signals suggests that the microemboli are gaseous in nature, that their production is pressure driven, and that cavitation is a possible cause. It is likely that mechanical heart valve design is responsible for the quantity of microemboli produced. It can be concluded that the mechanisms of HITS production are not affected by the composition of the inspired gas in their experimental conditions. Pacing increased the HITS count, but this increase was proportional to the heart rate. Pacing never influenced HITS production itself, as shown when the HITS count was expressed in HITS per 100 cycles. Alteration of contractility had a significant effect on HITS production [16]. In our study we investigated the relationship between exerciseinduced heart rate increase and the formation of microbubbles and HITS in mechanical heart valve implanted patients. Exercise increases myocardial 
contractility and dp/dt. We think that myocardial contractility and heart rate increase influences HITS and microbubble formulation.

We did not perform any neurocognitive tests during this study. Future studies on the clinical relevance of HITS in patients following mechanical heart valve replacement will need to focus on neurofunctional tests to shed additional light on highintensity transient signals.

In conclusion, in our study, we found that microbubbles increased as the heart rate increased and more HITS were propelled to the cerebral circulation. As shown before, HITS can alter cognitive functions. Therefore heart rate control (e.g. via $\beta$-blockers) is essential in mechanical heart valve patients to protect neurocognitive functions.

\section{References}

1. Spencer MP, Campbell SD, Sealey JL, Henry FC, Lindbergh J. Experiments on decompression bubbles in the circulation using ultrasonic and electromagnetic flowmeters. Occup Med 1969; 11: 238-44.

2. Spencer MP, Lawrence GH, Thomas GI, Sauvage LR. The use of ultrasonics in the determination of arterial aeroembolism during open heart surgery. Ann Thorac Surg 1969; 8: 489-97.

3. Geiser T, Sturzenegger M, Genewein U, Haeberli A, Beer JH. Mechanism of cerebrovascular events as assessed by procoagulant activity, cerebral microemboli, and platelet microparticles in patients with prosthetic heart valves. Stroke 1998; 29: 1170-7.

4. Georgiadis D, Wenzel A, Lehmann D, et al. Influence of oxygen ventilation on Doppler microemboli signals in patients with artificial heart valves. Stroke 1997; 28: 2189-94.

5. Rambod E, Beizaie M, Shusser M, Milo S, Gharib M. A physical model describing the mechanism for formation of gas mirobubbles in patients with mitral mechanical heart valves. Ann Biomed Eng 1999; 27: 774-92.

6. Jacobs A, Neveling $M$, Horst $M$, et al. Alterations of neuropsychological function and cerebral glucose metabolism after cardiac surgery are not related only to intraoperative microembolic events. Stroke 1998; 29: 660-7.

7. Pettelot G, Baudouy M, Morand P. Detection of cardiac embolism by transcranial Doppler [French]. Ann Cardiol Angeiol (Paris) 1996; 45: 34-8.

8. Rambod E, Beizai M, Sahn DJ, Gharib M. Role of vortices in growth of microbubbles at mitral mechanical heart valve closure. Ann Biomed Eng 2007; 35: 1131-45.

9. Dittrich R, Ringelstein EB. Occurrence and clinical impact of microembolic signals during or after cardiosurgical procedures. Stroke 2008; 39: 503-11.

10. Sylivris S, Levi C, Matalanis G, et al. Pattern and significance of cerebral microemboli during coronary artery bypass grafting. Ann Thorac Surg 1998; 66: 1674-8.

11. Grogott HP, Mackensen FB, Grigore AM, et al. Postoperative hyperthermia is associated with cognitive dysfunction after coronary artery bypass graft surgery. Stroke 2002; 33: 537-41.

12. Braekken SK, Russell D, Brucher R, Svennevig JL. Incidence and frequency of cerebral embolic signals in patients with a similar bileaflet mechanical heart valve. Stroke 1995; 26: $1225-30$
13. Gencbay M, Degertekin M, Basaran Y, et al. Microbubbles associated with mechanical heart valves: their relation with serum lactic dehydrogenase levels. Am Heart J 1999; 137: 463-8.

14. Kleine P, Perthel M, Hasenkam JM, Nygaard H, Hansen SB, Laas J. Downstream turbulence and high intensity transient signals following aortic valve replacement with Medtronic Hall or St. Jude Medical valve substitutes. Eur J Cardiothorac Surg 2000; 17: 20-4.

15. Droste DW, Hansberg T, Kemény V, et al. Oxygen inhalation can differentiate gaseous from nongaseous microemboli detected by transcranial Doppler ultrasound. Stroke 1997; 28: 2453-6.

16. Deklunder G, Lecroart JL, Lapeyre D, et al. Effects of myocardial contractility on microemboli production by mechanical heart valves in a bovine model. Tex Heart Inst J 2000; 27: 236-9. 